\title{
A Descriptive Model of the Designer's Problem-Solving Activity During the Later Phases of the Mechanical Engineering Design Process
}

\author{
Damien Motte \\ Division of Machine Design, \\ Lund Institute of Technology \\ at Lund University \\ damien.motte@mkon.lth.se
}

\author{
Per-Erik Andersson \\ Division of Machine Design, \\ Lund Institute of Technology at \\ Lund University \\ per-erik.andersson@mkon.lth.se
}

\author{
Robert Bjärnemo \\ Division of Machine Design, \\ Lund Institute of Technology \\ at Lund University \\ robert.bjarnemo@mkon.lth.se
}

\begin{abstract}
Many specific and precise methods that support the mechanical engineering designer's work during the conceptual design phase exist, while only a few general methods address the embodiment design and detail design phases. Our study presents the pattern of the designer's problem-solving activity during the later phases of the design process. This model is intended to serve as a basis for further development of tools and methods directly oriented towards the designer at work in these stages of the design process. The descriptive model presented here is developed through observations of six designers at work in controlled experiments, and follows a previous study published elsewhere.
\end{abstract}

\section{Introduction}

Numerous methods, based on a theoretical approach or on best practices, are dedicated to the process of mechanical engineering design, or design for short. These methods aim at optimizing the designer's activity of creating and developing an artifact in terms of costs, quality and time, by supporting his or her design activity. The pattern that underlies these methods is that their rigorous and rational application should naturally lead to a satisfying solution. The paradox is that little is actually known about the designer who carries out and is central to all of these methods. The designer is often considered as rational, skilled and with a huge amount of knowledge, but is this assumption relevant for the development of methods that support the design activity?

A growing number of research studies have been dedicated to how the designer actually thinks and acts. Based on findings in the field of cognitive sciences, these works have been mapping the range of skills and limitations a designer possesses with the aim of im- proving design methodologies. A whole body of knowledge is emerging, and several special issues of engineering design journals (like Design Studies vol. 18(4), vol. 19(4), vol. 20(5), vol. 21(5), Automation in Construction vol. 7(2/3)) and conferences (Design Thinking Research Symposia, Creativity and Cognition, International Conference on Design Computing and Cognition) testify to the importance of this issue. However, most of the studies reported concern the conceptual phase of the design process. Creativity is indeed central at this stage, and the "outputs" of this activity constitute the less "controlled" part of the design process. Moreover, the decisions taken at this phase are decisive for the pursuit of the product development project.

However, the embodiment design and detail design phases are still important due to the time they consume, the costs they generate and their importance for the quality of the product-to-be. Moreover, even if the working principle of the product is known and the design problem thus well defined, creativity is still required especially during the synthesization parts of these phases, when the product architecture, and the embodiment, "form" and shape of the product still do not exist. A deeper understanding of the designer's activities in the later phases of the design process is therefore needed.

The purpose of this paper is to present a descriptive model of the designer's problem solving activity during the embodiment design and detail design phases, through observation of the basic cognitive tasks fulfilled by the designer. This follows a preliminary study published elsewhere [1]. This paper also presents a refinement of the set of categories used for the analysis of the problem-solving process.

The first part of this contribution presents the background: purpose and framework of the study. The second part will discuss the theoretical limitations that the modeling of the design activity as a problem- 
solving process implies. The main findings on the cognitive aspects of problem solving in design are also presented. The third and fourth parts will reiterate the methodology used for this study, present the set of categories developed and used for the analysis of the problem-solving process, and the preliminary results of [1]. The descriptive model of the designer's problem-solving activity is then presented and discussed. Finally, future efforts needed to complete this investigation are proposed.

\section{Purpose and framework of the study}

A huge amount of methods have been developed to support the early phases of the design process, which are often precise and rigorous. They do not only 1) allow the designer to structure and plan his or her work, 2) they also consider the designer's limitations by supporting him or her to avoid becoming lost in the huge amount of information which has to be handled, they enhance the designer's creativity, help the designer to prioritize the design activities, etc. 3) They also take into account that teamwork in the design process is a requirement.

On the other hand, design process methods only partly take into account all of these three characteristics when they deal with the later phases of the design process. The process of design (considering the characteristics mentioned above) during the embodiment design and detail design phases is sometimes considered as a less complex activity than the conceptual design phase - in the sense that the task is more well defined - and thus only roughly developed (see e.g. [2], p. 16). Others, like [3] (p. 201) and [4] (p. 136), present a more detailed procedure, which rather concerns the planning of the different design activities for the whole technical system, thus fulfilling the first characteristic presented above, than helps the designer in his or her daily activity. [5] (p. 185-186) claims that it is impossible to have a more "step-by-step process", at least in the early part of the embodiment design phase. In [6], there is no constraining procedure; the author allows the designer the freedom to even switch between the conceptual design and the other phases according to the designer's needs and priorities. Thus the design process is supported partly by the designer's experience. [4] presents a "structure of possible activities in the design process" (p. 135). This structure is supposed to be the same for all the phases of the design process (conceptual design, embodiment design and detail design). If it gives an insight into the activities that the designer may need to perform, there are few guidelines on how to structure these activities.

Thus, during the embodiment design and detail design phases, the designer develops and structures the work, and chooses the techniques, tools and standards needed, based almost exclusively on the designer's experience and education. There is a need to develop methodic support to the designer that structures the design activities during the embodiment design and detail design phases.

To that end, a study has been undertaken whose approach is to observe the designer at work. This should lead, helped by the literature on design practice [7], to identifying the moments where the designer needs support and how this task can be organized. The designer's observations should lead, in turn, to the identification of "best practices" that can be exploited for the development of support. The designer is observed within a four-level framework, partly similar to the structural design process presented by Hubka in [4]: 1) the designer placed in his or her daily work environment; 2) the tactics and strategies applied during the whole embodiment and detail design activities (developed in [8]); 3) the operational, cognitive activities during design, especially problem solving (detailed here); 4) the basic cognitive elements: induction, deduction, abstraction, perception, pattern recognition, attention, intelligence, etc. (these elements are not design-specific, and thus are beyond the scope of the study reported here). Figure 1 presents the framework.

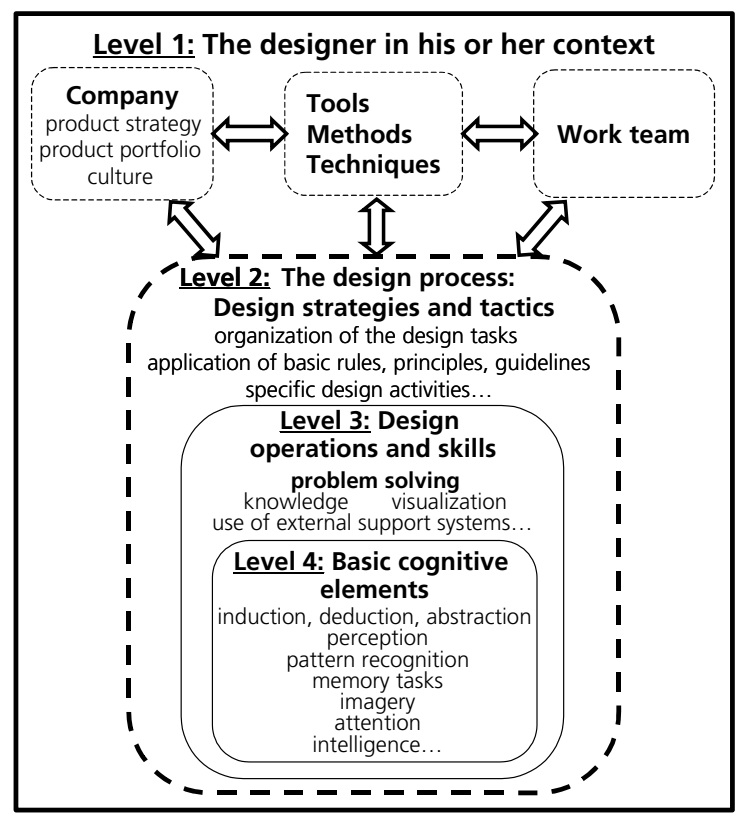

\section{Figure 1. The Four-Level Study Model of the Designer's Activities.}

In this paper, a refinement of the description of the problem-solving process during the embodiment design and detail design phases is presented. This means that the coding scheme necessary for the study of the designer that had been developed in [1] has been refined (see section 4); further aspects of the 
problem-solving process not undertaken in [1] are developed, namely, the search for information, the refinement of the problem and the evaluation moments.

\section{Problem-solving process in engineering design}

A design task is often considered as a problem to solve, and problem solving is often modeled as sequential. These views are used as work hypotheses of this study. Their limitations are given in the first part of this section. The second part presents the main findings on the cognitive aspects of problem solving in design

\subsection{Work hypotheses}

Our work on the problem-solving process is based on two hypotheses that are discussed in this section: that the problem-solving process is sequential, and that most design activities can be modeled as a problemsolving process.

First of all, the concept of "problem" needs to be clarified. Although there are as many definitions of a problem as there are authors, there is a consensus that a problem is a discrepancy between an observed state and a desired state, with no known solution (the observed state and desired state can vary with time). The problem-solving process is then the elaboration of a solution whose implementation suppresses the discrepancy.

The traditional view in the problem-solving literature is that of the "phase theorem", which means that a problem is solved rather sequentially. This idea was first developed by Dewey in 1910 [9], who proposed a five-step model: 1) a felt difficulty, 2) its location and definition, 2) suggestion of possible solutions, 4) development by reasoning of the bearing of the suggestion, 5) further observation and experiment leading to its acceptance or rejection. In the field of mechanical engineering design, as well as in any other field, the problem-solving processes are described in this way (see e.g. [4], [3] and [2]). As described in [10], "the descriptive facet of the [phase] theorem suggests that problem solvers follow a certain sequence of phases. Its prescriptive facet suggests that problem solvers are more likely to succeed if they follow a certain sequence of phases." (p. 48). However, though widely accepted, the validity of both the prescriptive and descriptive models is still questioned [10]. No study has so far been conclusive, and we do not even know if the problem-solving processes models in the literature represent the actual process-solving process or if they are "implicit schemata of how problems are, and should be, solved" [10] (p. 48).
The second hypothesis is that most activities in design can be modeled as a problem-solving process. This is a well and widely accepted assumption. Even Simon in [11] presents the problem-solving model: "intelligence", "design", and "choice" (which can roughly correspond to: "problem understanding", "solution generation, "evaluation-decision"), using the word design to describe the core of the problemsolving process. However, the assumption that the design activity is a problem-solving process has been recently challenged in [12]. Design is rather seen as containing problem solving, rather than being a special case of problem solving; the design problems should be seen as projects to handle with an infinite number of problems, rather than just problems. Design thus needs to be seen from another perspective. The rationale behind this claim is developed in [12]. The implications are that the modeling of a design activity as a problem-solving process may not be sufficient to describe it.

During the later phases of the design process, the design tasks are fortunately more well defined than in the conceptual design phase. Thus the last point has limited consequences for the study. The claim developed in [12] should nevertheless be investigated in further studies.

The validity of the sequentiality of the problemsolving activity is still discussed, but this model nevertheless has the advantage of being a powerful tool to describe problem-solving process observations. Thus we chose to rely on it.

\subsection{Cognitive aspects of problem solving}

The main findings from the literature, valid for both earlier and later phases of the design process, have been presented in a literature survey [7]. Here follows a summary of the main characteristics of human problem solving in design: early appearance and persistence of a kernel idea; design fixation (inclination to stick with early satisficing solutions); lack of flexibility in designer's thinking behavior; superficial assessment; subjective judgment.

\section{Method of investigation}

\subsection{Observations under controlled experi- ments}

The most widely used method to observe problem-solving activities in cognitive sciences is to perform laboratory-like experiments with verbal protocol analysis (VPA). Experiments allow a control over many parameters: here we wanted to focus solely on the design process, without external influence (see Figure 1), thus experimentation was the best way to 
Table 1. Categories of the Coding Scheme.

\begin{tabular}{|c|c|}
\hline Category & Description \\
\hline $\operatorname{Irp}$ & $\begin{array}{l}\text { Concerns the time segments where the subject asks the experimenter for complementary information on the prob- } \\
\text { lem itself. That is, the subject asks for information helping in the understanding of the problem, not for directly } \\
\text { developing a solution. }\end{array}$ \\
\hline $\mathrm{Sp}$ & Concerns the time segments where the designer reformulates, re-frames the problem (from [16] and [14]). \\
\hline Ep & Concerns the time segments where the subject evaluates the problem itself. \\
\hline Irm & $\begin{array}{l}\text { Concerns the time segments where the subject asks the experimenter for complementary information on mechan- } \\
\text { ics. That concerns formulas, models... }\end{array}$ \\
\hline $\mathrm{Sm}$ & $\begin{array}{l}\text { Concerns the time segments where the subject describes the solution in mechanical terms (force, moment; strain, } \\
\text { stress; buckling; etc.) }\end{array}$ \\
\hline Em & Concerns the time segments where the subject evaluates his or her mechanical model. \\
\hline Irs & $\begin{array}{l}\text { Concerns the time segments where the subject asks the experimenter for information that directly helps the syn- } \\
\text { thesis activity. It can be catalogues of components, of joints... }\end{array}$ \\
\hline Ss & Concerns the time segments where the subject creates the form and layout of the support. \\
\hline Es & Concerns the time segments where the subject evaluates his or her solution (layout, form, or the overall solution). \\
\hline Ird & Concerns the time segments where the subject asks the experimenter for information that helps in dimensioning. \\
\hline $\mathrm{Sd}$ & Concerns the time segments where the subject dimensions the artifact. \\
\hline Ed & Concerns the time segments where the subject evaluates the results of dimensioning. \\
\hline $\mathrm{D}$ & Concerns the time segments where the subject documents his or her work by a detail drawing. \\
\hline Eego & Concerns the time segments where the subject evaluates himself or herself. \\
\hline $\mathrm{O}$ & Concerns the time segments where the subject organizes his or her way of working. \\
\hline
\end{tabular}

control the information the designer had access to. Verbal protocol analysis is a technique developed by [15]: The subject is recorded while "thinking aloud", and his or her "thoughts" are then transcribed and analyzed with the help of a set of categories each describing a single action. Even if it is still a subject of controversy, "thinking aloud" (the subject says what he or she is thinking) has been the best technique so far in order to obtain a detailed description of a cerebral activity.

In this paper, we used the six experiments designed in [1]. The subjects were three students and three experts. Two students were seniors, one was a junior. All the experts had more than 20 years experience. One has always worked in industry, one always in academia, while the third had worked half in industry, half in academia.

The experimental procedure is given for information in the Appendix. The set of categories used to analyze the transcribed verbal protocol, also called coding scheme, is the development of the coding scheme developed in [1]. The coding scheme is provided in the next section.

\subsection{Developed coding scheme}

The coding scheme is presented in Table 1.

The model-coding scheme presented 7 categories [1]. It gave insights into the problem-solving activities performed by the subjects, especially solution development (see next section). For this paper, the activities that are addressed are the problem understanding activity, the search of information and particularly the evaluation moments. Thus the coding scheme has been extended to 15 categories.

The evaluation categories (Ep, Em, Es, Ed) were further analyzed following two dimensions: the type of evaluation, and the role of evaluation. The type of evaluation represents the way the evaluation is made: with or without criteria. When the evaluation was without criteria, then the type of evaluation was further divided between a qualitative type of evaluation (good, bad...) or binary type of evaluation (wrong/right). The role of evaluation addresses the aims of these evaluations: decision, reinforcement (or confirmation) of a decision, judgment, comparison between two sub-solutions, and control (or check). 
Table 2. Dimensions of the evaluation moments.

\begin{tabular}{|c|c|}
\hline Category & Description \\
\hline \multicolumn{2}{|c|}{ Types of the evaluation } \\
\hline $\begin{array}{l}\text { qu - qualitative } \\
\text { evaluation }\end{array}$ & $\begin{array}{l}\text { Concerns the evaluation moment where the designer qualitatively evaluates his or her solution (or the prob- } \\
\text { lem). Examples: "This looks strange", "my part is really clumsy", "I think I am satisfied with this de- } \\
\text { sign"... }\end{array}$ \\
\hline $\mathrm{r}-$ right/wrong & $\begin{array}{l}\text { Concerns the evaluation moment where the designer makes a "right or wrong" evaluation, without any } \\
\text { criteria (note that this kind of evaluation is not always followed by a decision.) }\end{array}$ \\
\hline $\mathrm{cr}-$ criterion & Concerns the evaluation moment where the designer makes an evaluation with the help of a criterion. \\
\hline \multicolumn{2}{|c|}{ Roles of the evaluation } \\
\hline $\mathrm{d}$ - decision & $\begin{array}{l}\text { Concerns the evaluation moment where the evaluation leads to a decision to continue the development of a } \\
\text { solution or not. The evaluation moment and the decision could rarely be separated, the decision being taken } \\
\text { implicitly. }\end{array}$ \\
\hline $\mathrm{r}$ - reinforcement & Concerns the evaluation moment where the evaluation is a reinforcement of a previous decision. \\
\hline $\mathrm{j}$ - judgment & $\begin{array}{l}\text { Concerns the evaluation moment where the evaluation is a judgment of a solution without any subsequent } \mathrm{t} \\
\text { decision. }\end{array}$ \\
\hline $\begin{array}{l}\text { comp - compari- } \\
\text { son of solutions }\end{array}$ & Concerns the evaluation moment where there is a comparison between two sub-solutions. \\
\hline $\begin{array}{l}\mathrm{c}-\text { check } \\
\text { (control) }\end{array}$ & $\begin{array}{l}\text { Concerns the evaluation moment where there is a control or check over what has been done so far. It is } \\
\text { different from the reinforcement in the sense that the control episode does not concern a decision, but rather } \\
\text { the design activity and its result(s). }\end{array}$ \\
\hline
\end{tabular}

These dimensions are presented in Table 2. The category Eego, representing the moments where the designers evaluated themselves, was not the object of further investigation. This category is included in the coding scheme because this action has been observed in the majority of the experiments (5 out of 6).

The verbal protocol of the experiments has been segmented in elementary problem-solving episodes to which a category of the coding scheme was assigned. This analysis served as basis for the interpretation of the experiments. The results of the previous study relevant for this paper are summarized in the next section, while the results of this study are developed in section 6 .

\section{Previous results: general problem- solving strategy and solution development}

As mentioned before, we accepted as our point of departure the general phase theorem. The different problem-solving models present in the literature are not very distinct from each other [10]. Thus we adopted the model developed by Simon [11], which corresponds to the three core steps of the model introduced in our field by Hubka [13], and which is further developed by Eder [14].
The model proposed, then, has the form "task understanding / solution generation / evaluation". Our assumption was that generating a manifold of solutions was mainly a necessity for the conceptual design phase. This model emphasizes novelty and creativity during the process, which might be not necessary during the embodiment design and detail design phases. Thus we focus mainly on solution generation.

Our first study [1] partly confirmed our assumption. All the designers observed presented the same pattern of a general problem-solving strategy: they quickly understood the problem, developed no more than 2 alternatives, rapidly selected one of them and then lengthily studied and developed this alternative (by dimensioning or not - two students did not dimension, two experts dimensioned with only the help of their experience). The generation (or development) of the solution was interplay between synthesis and mechanic modeling. Synthesis, in short, was the activity of creating the solution, while mechanical modeling was the modeling of this solution. Synthesis usually preceded mechanical modeling. Finally, the detail-drawing episode, the first moment where the designers were confronted with real proportions and measures, was always the case of coming back to synthesis. 


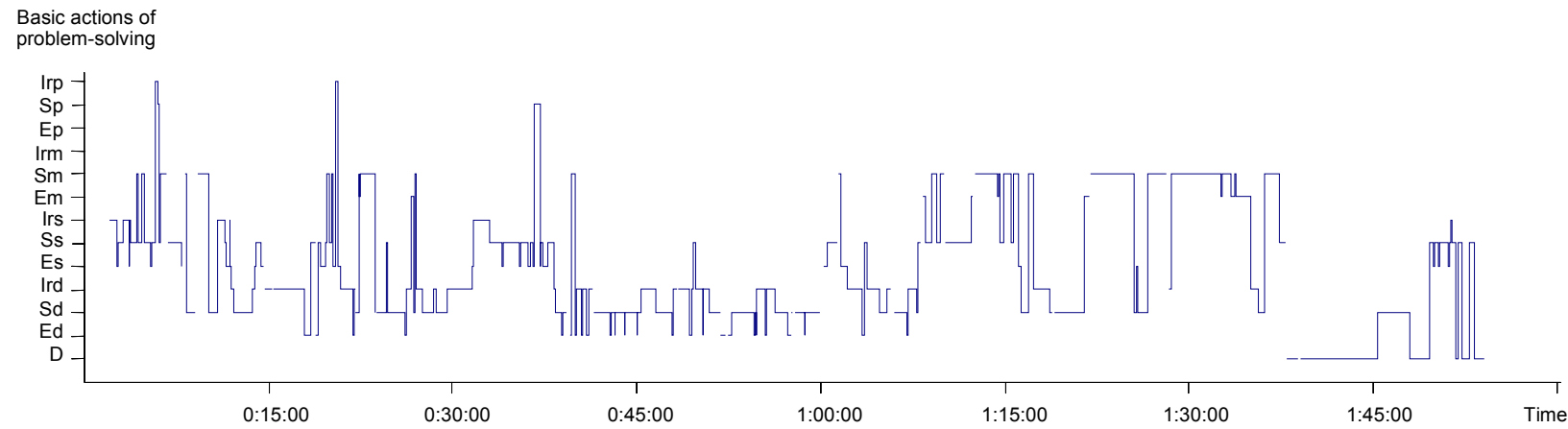

Figure 2. Problem-solving activity of an expert.

\section{Results and Discussion}

In this section, we present and discuss the main findings concerning the episodes of information search, problem understanding and evaluation, before we present a descriptive model of the problem-solving process during the embodiment and detail design phases developed from this exploratory study.

The pattern of activities of one expert is given as an illustration in Figure 2. The episodes of selfevaluation Eego and organization $\mathrm{O}$ have been removed from the diagram in order to facilitate its reading, and because these episodes were not directly constitutive of the problem-solving process.

\subsection{Information search}

Contrary to our previous study, we distinguished between the aims of the information search. For all but two designers (one expert and one student), the time dedicated to information search on the problem (Irp) did not exceed $30 \mathrm{sec}$. This is really a short time, and it shows that designers do not question the problem, as they would do for conceptual design [17]. From the expert, as could be expected, no time was dedicated to the search for information that would help the development of a mechanical representation of the solution (Irm). Two students needed that information, one junior and one senior, but the senior needed a model for buckling, which is quite specific. In fact, the solutions designed by the experts were relatively easier to model, thus requiring less information (and less time) [1]. There was not much difference between the designers studied concerning information search for the synthesis activity (the two experts that dimensioned without performing any calculation took less time, however). The search for information concerned the search for standard components. The students took more time studying the standard mountings of the hydraulic cylinder, while they did not bother very much looking for standard components for their design. Finally, the time dedicated to search information for dimensioning cannot be compared: some designers did not dimension at all, while others dimensioned with the help of their experience. The Ird episodes were often more structured than others. Although it is not visible in our coding scheme, we have observed that the designers sometimes used criteria in order to search this information, searched among a large amount of information and then rigorously selected the information with the help of the criteria. Generally, it has been observed that the more well defined the problem is, the more rigorous are the designers in their tasks.

In summary, although designers spent a long time searching information (from $15 \%$ to $35 \%$ of their time), this information was oriented towards the information they needed and not towards a better understanding of the problem.

\subsection{Problem understanding}

As mentioned earlier, information search for problem understanding is negligible. The time spent for reframing or reformulating the problem is not much either: around $30 \mathrm{sec}$. to $1 \mathrm{~min}$. (less than $2 \%$ of the experiment time). Some designers did not question the problem at all. The designers did not go beyond the brief they received. One could argue that this behavior is due to the experimental, even "scholarly" context of the task (one to two hours in front of an experimenter). But in [17], experts, under the same conditions, had to fulfill a conceptual design task; most of them asked more than needed in order to get an overall idea of the task and not to forget important points. The importance of the problem clarification is emphasized by the literature (see e.g. [3], [14], [17]). Thus this is a point that should be recommended to the designer even at a later stage of the design process. 


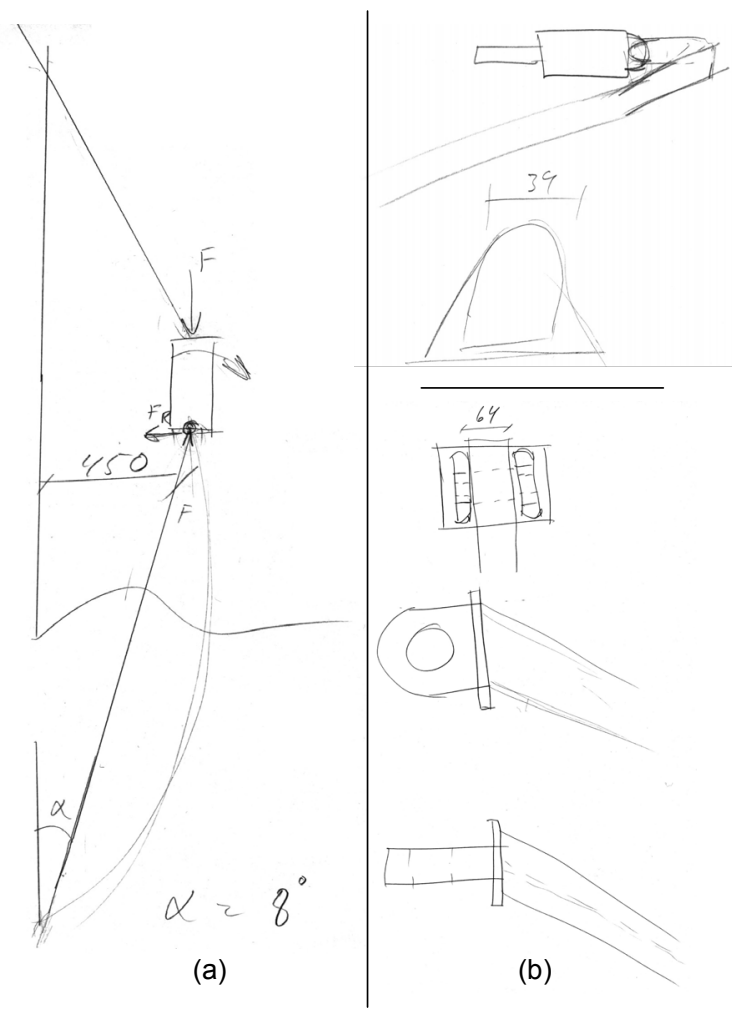

Figure 3. (a) First sketch of a student; (b) Concretization of the solution: interface problem.

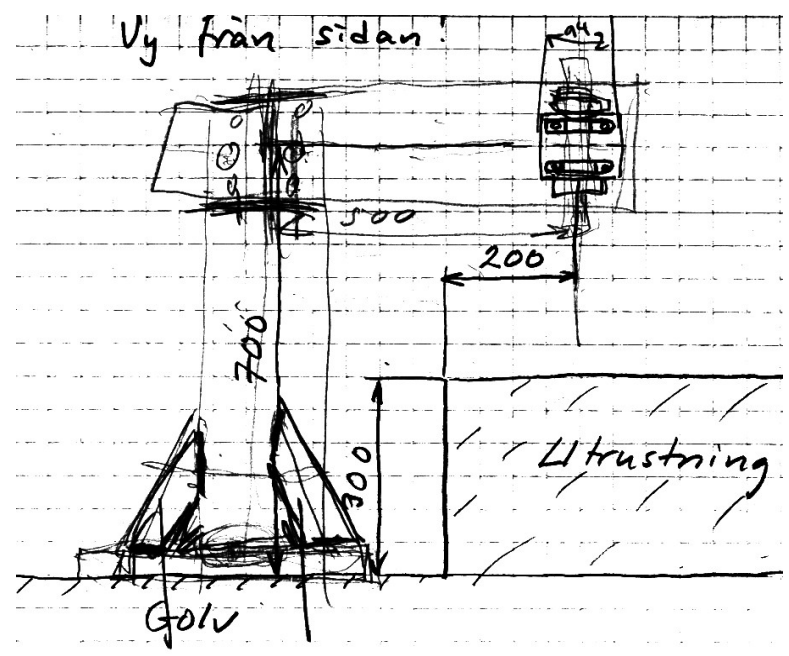

Figure 4. First sketch of an expert.

To what extent the designers should spend time on this activity remains unclear, however. Should they question the whole problem? What has been observed is that the designers are really focused on the development of the solution. This makes their work very effective. For real problems, the time given to the designer is not as vague as for conceptual design; the results of their work can be quantified and evaluated; thus the designer has to be effective and focused. The question remains to determine in which proportion of the working time the problem must be reformulated, and how it must take place in the overall design process.

The junior student did indeed spend significantly more time on problem reformulation. However, what has been observed is that his behavior was what in [18] is called "adhocism". At many times, the student did not actually try to understand the problem, but rather tried to reformulate it so that it would fit the technical system he had developed and the knowledge he had. This phenomenon must be taken into account for further development of this issue.

\subsection{Evaluation episodes}

It as been decided, to avoid overly expanding our coding scheme, that the evaluation episodes comprise the evaluation, decision, verification, and check (control) episodes. Recently, in [14], emphasis has been placed on the action of "reflecting over". A control on our coding showed that this step had been partly included in the evaluation episodes, partly in the solution development episodes ( $\mathrm{Sm}, \mathrm{Ss}, \mathrm{Sd})$.

The evaluations of the problem-understanding episodes were very few; most of the experts did not even have one single episode, like the one presented in Figure 2. This is a logical consequence of the small amount of time spent on problem understanding, as developed in the last section. Otherwise, most of the evaluation episodes Ep were qualitative, and their role was that of judgment.

It has been mentioned in [8] that the experts applied the basic rule of simplicity developed in [3], but most often only during the first half of the experiment. Then their design became slightly more complex. This explains why there are an increasing number of evaluation episodes following the mechanical modeling moments during the experiments (in about $30 \%$ of the cases for the experts). The students had a slightly higher percentage. These evaluation episodes mainly concerned decision-making episodes (d), and they were always without criteria (half were qualitative "qu", half were "r").

The evaluation episodes for synthesis (Es) were the most frequent for every designer, followed immediately by the evaluation episodes for dimensioning (Ed) for the designers who performed calculations for dimensioning. The purpose of these evaluations comprised all the evaluation roles described above $(\mathrm{d}, \mathrm{j}, \mathrm{r}$, comp, c). Decision was the major cause of evaluation, followed by judgment of the solution, comparison between solutions and sub-solutions and finally control (although to a smaller extent than for Em and Ed). 


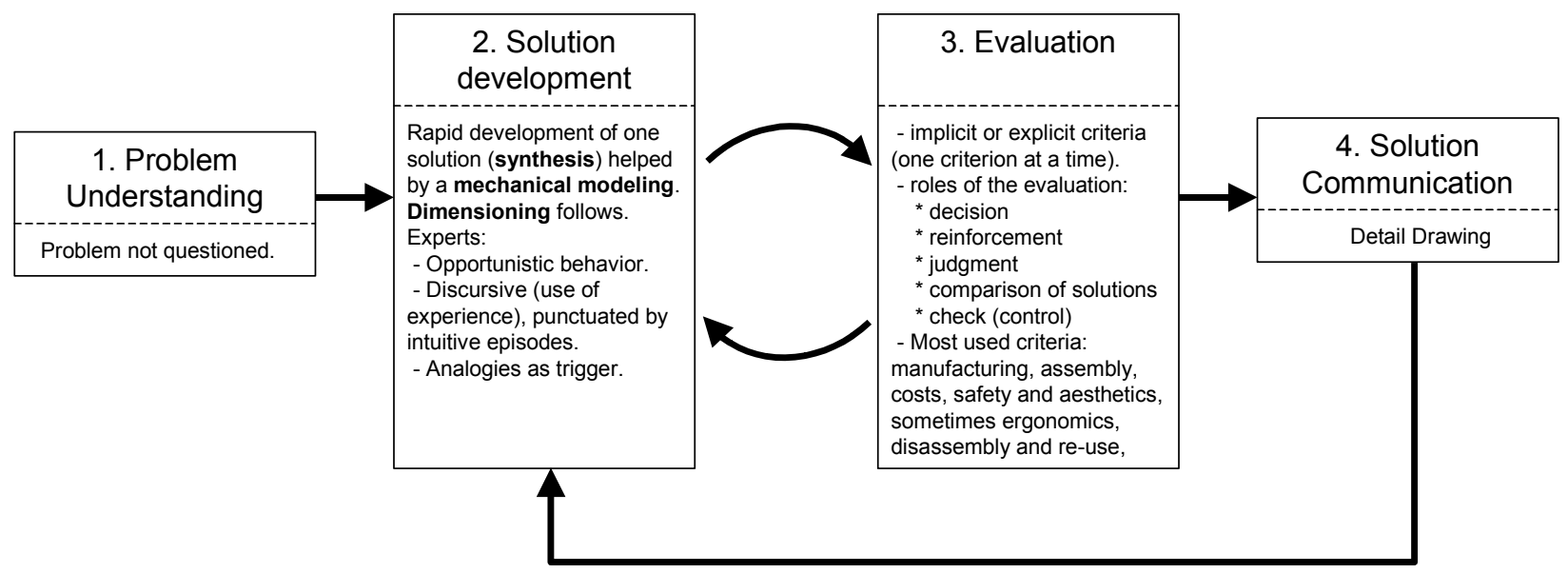

Figure 5. Descriptive problem-solving activity model.

Evaluations following dimensioning obviously occurred when the designers explicitly calculated the dimensions. The decision was then, following the result, to go on or come back to the dimensioning. Results of dimensioning were not used to compare two alternatives (at least it did not appear explicitly in the verbal protocol), mainly because the solution alternative was already chosen during the synthesis episode. A minority of decisions was based on criteria. This is due to the fact that the criteria did not need to be given explicitly, the task often being to see whether the component chosen would fulfill the mechanical constraints.

The Eego episodes were relatively rare; they appeared once or twice during most experiments. At this moment, the designer questions his or her own capacity to solve the problem or sub-problem. This moment does not directly concern the problem-solving process, but it showed that the designers are also making a statement about themselves. However, it is difficult to interpret this further. It may be a way of challenging oneself, encouraging oneself to perform better - but it cannot be excluded that this was triggered by the presence of the experimenter.

All the evaluation moments, although representing around $2 \%$ to $10 \%$ of the experiment time, represented often the majority of the number of episodes $(10 \%$ to $25 \%)$. Decision was the main consequence of the evaluation moments (more than the half of the cases), followed by judgment. Between $60 \%$ and $80 \%$ of the evaluations are taken without explicit criteria. Half of them are of type "qu", half of type "r". This does not mean, however, that the designers do not have any criteria. It is rather the state of affairs that, in the case "qu" and " $r$ " they use criteria that are intrinsic to their experience [19].
The nature of the criteria used has been described in [8]: the experts mostly take into account manufacturing, assembly, costs, safety and aesthetics, sometimes ergonomics, disassembly and re-use, but most of the factors presented in [3] p. 205 are neglected. The students were not much concerned about factors influencing the design process.

\subsection{A refined model of the problem-solving process during the embodiment and detail design phases}

As Figure 2 shows, the designer does not follow the steps of problem solving as they are generally prescribed. The designer makes mistakes, needs to sometimes return to problem understanding, has rather an opportunistic way of solving the problem: going very deep into detail when he or she has the knowledge required. And this has been proved to work better concerning the embodiment design for the following reason: by choosing the details of the artifact very early in the process, the designer very quickly apprehends the problem of interfaces between parts [1]. The student, who remains at a higher level of abstraction, needs then to introduce non-standard components, which augments the number of manufacturing and assembly operations (it is worth noticing that the experiment concerned an artifact to be produced in only a few numbers. There may be other conclusions for a mass-produced artifact). This is visible in Figure 3 and Figure 4, which illustrate the differences between an expert and a student.

The particularities of the problem-solving activity have been stressed throughout this paper. However, the generic prescriptive model (problem understanding, generation of solutions, evaluation-decision) still constitutes the core of the problem-solving activity. 
What differs is the content of these moments. The descriptive model of problem solving during the later phases of the design process is presented below. It synthesizes the details of each phase as presented in [1] and in the preceding section). The model is represented Figure 5.

1) Problem understanding: The problem is understood quickly because it is well defined. The designers do not question the stated problem, nor do they come back to it during design.

2) Solution generation (development): The designer rapidly develops one solution (synthesis) helped by a mechanical modeling of the solution. Dimensioning follows. Interactions between the mechanical modeling activity and the dimensioning activity have seldom been observed. This process of actions is represented Figure 6. The sequence of transitions between the actions that has been most often observed follows the order i-iv. When material, components and joints are chosen from the beginning, the design process goes more rapidly. The sub-problems are generally treated separately and deeply by the experts. Nevertheless, the first embodiment is generally complete (all the parts that constitute the embodiment are present). The behavior of the expert during synthesis was discursive (use of experience), punctuated by intuitive episodes, in the form of "illumination" in accordance with the model of [20]. Designers used sometimes analogies as triggers. The students used a case-driven analogy (remembered a similar previous case), while the experts used more abstract comparisons, triggering solution ideas (schemata-driven analogy). These observations seem to show that the model of spontaneous analogizing developed in [21] for the conceptual design phase is also relevant for the later design phases.

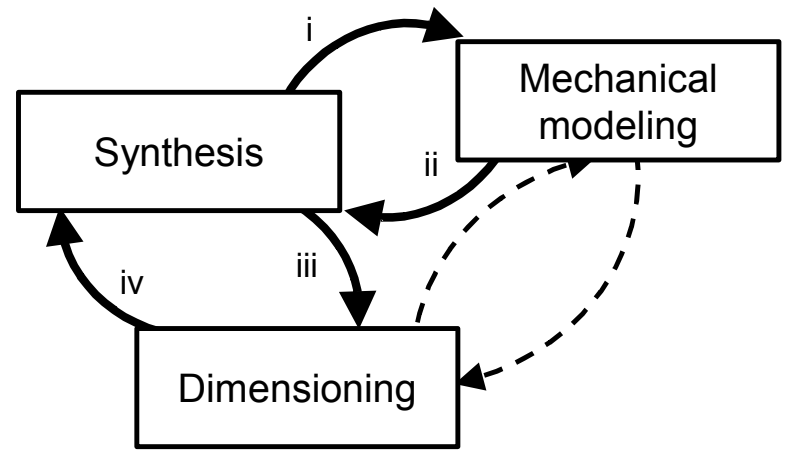

Figure 6. The process of actions during solution development.

3) Evaluation: Evaluation is made by implicit or explicit criteria. In this case, only one criterion is used. The evaluation moments are numerous, and made at any moment of the design, that is, the designer constantly checks the accuracy of his/her work.

4) Communication of the solution [14]: In our case, it is the detail drawing. The detail drawing passively plays the role of control of the solution (because all specifications must be present). All designers face problem with proportions and measures not taken into account during the solution development, and the designers must come back to synthesis.

\section{Conclusion}

We have presented a refined coding scheme that allows the analysis of the problem-solving activity during the embodiment design and detail design phases. This results in a model that describes in detail the problem-solving activity of the designer at this stage.

This model now needs to be complemented by the other levels of study presented in section 2: 1) the designer placed in his or her daily work environment (to be carried out);2) the tactics and strategies applied during the whole embodiment and detail design activities (developed in [8]). The next step will be the validation of the most important points. With only six experiments, this study was indeed only explorative in nature.

The descriptive model of the design process must then be utilized in order to support the design activity. For that purpose, the sets of actions of the designers need to be interpreted in terms of weaknesses and strengths. The weaknesses should be propped up, while the strengths should be included in a more specific prescriptive model for the embodiment design and detail design phases.

Finally, there is a need to remain aware of different views on the design activity (other than the problem-solving view, like the one presented in [12]) that could give supplementary information on the improvement of the design process.

\section{References}

[1] D. Motte, P.-E. Andersson, and R. Bjärnemo, "Study of the Designer's Cognitive Processes During the Later Phases of the Engineering Design Process", Proceedings of the Design 2004, Dubrovnik, 2004, pp. 421-428.

[2] K. T. Ulrich, , and S. D. Eppinger, Product Design and Development ( $3^{\text {rd }}$ Ed.), McGraw-Hill, London, 2003.

[3] G. Pahl, and W. Beitz, Engineering Design - A systematic approach ( $2^{\text {nd }}$ Rev. Ed.), Springer, London, 1996. [4] V. Hubka, and W. E. Eder, Design Science, Springer, London, 1996.

[5] D. G. Ullman, The Mechanical Design Process ( $2^{\text {nd }}$ Ed.), McGraw-Hill, New-York, 1997. 
[6] S. Pugh, Total Design - Integrated Methods for Successful Product Engineering, Addison-Wesley, Reading, MA, 1990.

[7] D. Motte, and R. Bjärnemo, "The Cognitive Aspects of the Engineering Design Activity - A Literature Survey", Proceedings of the TMCE 2004, Lausanne, 2004.

[8] D. Motte, P.-E. Andersson, and R. Bjärnemo, "A Study of the Mechanical Designer's Strategies and Tactics During the Later Phases of the Engineering Design Process", Proceedings of the DTM/ASME 2004, Salt Lake City, 2004 (accepted).

[9] J. Dewey, How we Think, Heath \& Co, Boston, 1910. [10] R. Lipshitz, and O. Bar-Ilan, "How Problems are Solved: Reconsidering the Phase Theorem", Organizational Behavior and Human Decision Processes, vol. 65(1), 1996, pp. 48-60.

[11] H. A. Simon, The New Science of Management, Harper, New York, 1961.

[12] A. Hatchuel, "Towards Design Theory and expandable rationality: The unfinished program of Herbert Simon", Journal of Management and Governance, vol. 5(3/4), 2001, pp. 260-273.

[13] V. Hubka, Theorie der Konstruktionsprozesse (Theory of Design Processes), Springer, Berlin/Heidelberg, 1976.

[14] W. E. Eder, and V. Hubka, "Reflections about Reflective Practice", Proceedings of the Design 2004, Dubrovnik, 2004, pp. 177-182.

[15] K. A. Ericsson, and H. A. Simon, Protocol Analysis: Verbal Report as Data (Rev. Ed.), MIT Press, Cambridge, MA, 1993.

[16] P. Lonchampt, G. Prudhomme, and D. Brissaud, "Engineering Design Problem in a Co-Evolutionary Model of the Design Process", Proceedings of the Design 2004, Dubrovnik, 2004, pp. 361-366.

[17] R. Eisentraut, J. Gunther, “ Individual styles of problem solving and their relation to representations in the design process”, Design Studies, vol. 18(4), 1997, pp. 369-383.

[18] B. Bender, L. Blessing, "On the Superiority of Opportunistic Design Strategies during Early Embodiment Design", Proceedings of the Design 2004, Dubrovnik, 2004, pp. 117-122.

[19] N. Bonnardel, T. Sumnar, "Supporting Evaluation in Design”, Acta Psychologica, vol. 91, 1996, pp. 221-244.

[20] G. Wallas, The Art of Thought, Watts, London, 1926 (reprinted 1949).

[21] L. Ball, T. Ormerod, N. Morley, “ Spontaneous Analogising in Engineering Design: A Comparative Analysis of Experts and Novices", Proceedings of the Design Thinking Research Symposium 6, Sydney, 2003.

\section{Appendix: The experimental procedure}

The experiment, for each of the subjects, lasted for two hours. Each experiment took place in an isolated room. The subject was face-to-face with an experimenter. To the left of the subject, a video camera, manipulated by a second experimenter, recorded the sequence, following the focus and the actions of the subject.

After a short exercise in practicing thinking aloud, the mission statement was delivered to the designer. The subject had to design and dimension a support device for a hydraulic piston that had to be fixed to the ground. The piston, guided laterally, had to resist an axial force of $90 \mathrm{kN}$. Under the piston, an installation was located on the ground. The support was to be located by the side of this installation (see Figure 7). The specifications of the piston were given in the assignment. This design task, then, was relatively well defined, and should correspond to what can be expected from a similar case in industry. Intentionally, the form-giving aspect was not very complex, so that the subjects had time for both synthesis and dimensioning. The designers were expected to produce a final sketch of the technical system. Finally, there was a short interview in which the subjects were asked to evaluate their design and the experiment.

The assignment has most of the characteristics of an embodiment and detail design task.
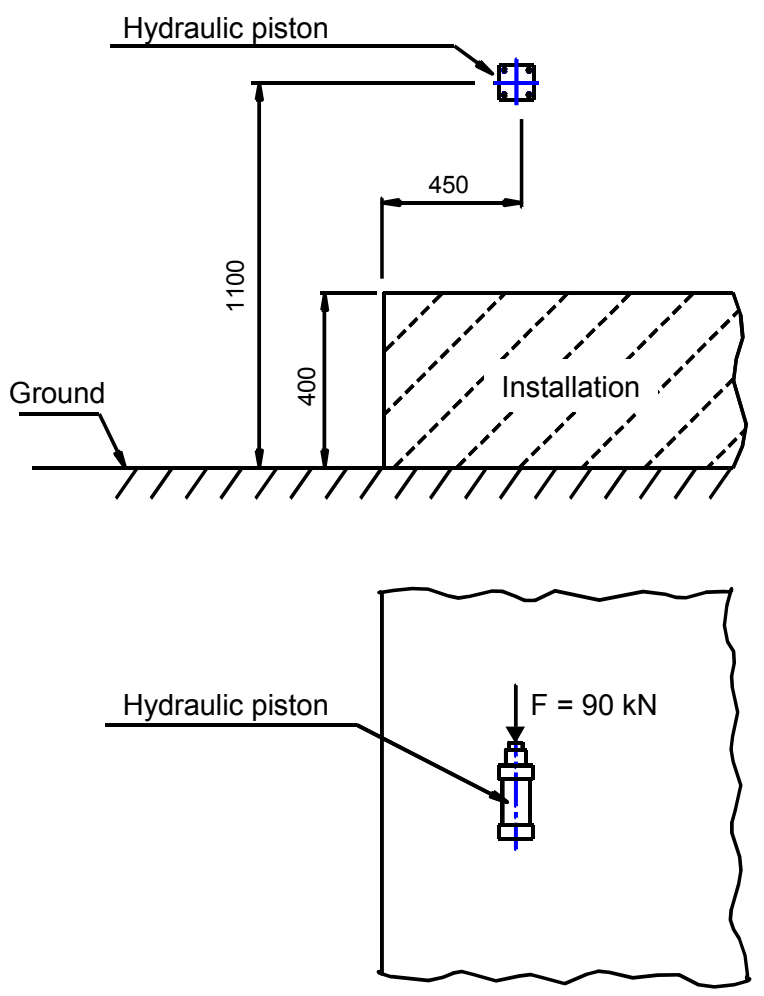

Figure 7. Sketch of the problem delivered with the assignment [1]. 